\title{
ON CYCLIC VECTORS OF THE BACKWARD SHIFT'
}

BY R. G. DOUGLAS, H. S. SHAPIRO AND A. L. SHIELDS

Communicated by P. R. Halmos, August 1, 1966

The forward shift operator $U$ (that is, multiplication by the independent variable) on the space $H^{2}$ of the unit circle has been much studied. In particular it is known that a vector $f$ is cyclic (that is, $\left\{U^{n} f\right\}(n \geqq 0)$ spans $\left.H^{2}\right)$ if and only if it is an outer function; that every invariant subspace is cyclic; and that two vectors generate the same invariant subspace if and only if they have the same inner factor (see [1, Chapter 5]).

Much less is known about the adjoint operator $U^{*}$ (the backward shift). The only published result seems to be in [2] where it is shown as a by-product of another investigation that a transcendental entire function is a cyclic vector for $U^{*}$. The problem of describing the cyclic vectors for $U^{*}$ was also posed by $\mathrm{D}$. E. Sarason at the Conference on Analytic Functions, held in Lexington, Kentucky, in May 1965. Both Helson and Sarason have a number of unpublished results on this problem; in particular several of the following theorems were known to them. In this note we indicate some of the results that we have obtained; full details will appear elsewhere.

Let $C$ denote the set of functions in $H^{2}$ of the unit circle that are cyclic vectors of $U^{*}$; let $N$ denote the set of noncyclic vectors.

TheOREM 1. $N$ is a dense vector subspace of $H^{2}$, and $N+C \subset C$.

Let $Q$ denote the set of functions on the unit circle that are equal almost everywhere to the quotient of two inner functions.

Theorem 2. A function $f$ in $H^{2}$ is in $N$ if and only if signum $f^{2}$ is in $Q$.

Corollary. $(N \cdot N) \cap H^{2} C N ;(C \cdot N) \cap H^{2} C C$; if $f \in C$ and $1 / f$ $\in H^{2}$, then $1 / f \in C$.

Here $N \cdot N$ denotes the set of all products $f g$ with $f, g \in N$.

CoRollary. A function $f$ in $H^{2}$ is in $C$ if and only if its outer factor is in $C$.

Corollary. If $f=\sum a_{n} z^{n} \in N$ then $\sum\left(\operatorname{Re} a_{n}\right) z^{n} \in N$.

Theorem 3. If $\int \log |\operatorname{Re} f| d \theta=-\infty$, then $f \in C$.

1 This research was supported in part by the National Science Foundation. 
Corollary. $C+C=H^{2}$.

For $f \in H^{2}$ let $K_{f}$ denote the invariant subspace of $U^{*}$ generated by $f$ (that is, the span of $\left.\left\{U^{* n} f\right\}(n \geqq 0)\right)$.

THEOREM 4. Every invariant subspace of $U^{*}$ is cyclic, that is, of the form $K_{f}$ for some $f$.

LEMma. Let $f \in H^{2}$, then $K_{f}$ is finite dimensional if and only if $f$ is a rational function.

Let $f_{r}(z)=f(r z)(0 \leqq r \leqq 1)$.

THEOREM 5. If $f \in C$, then $f_{r} \in C(0<r \leqq 1)$; if $f \in N$, then either $f_{r} \in C$ for all $r(0<r<1)$ or $f$ is a rational function.

COROLlaRy. If $f$ is analytic in a larger disc $(|z|<1+\delta)$, then either $f$ is cyclic or $f$ is a rational function.

A function $f$ is analytic in a larger disc if and only if its Taylor coefficients are exponentially small. From this point of view the hypothesis in the corollary cannot be weakened. Indeed, if $\left\{\epsilon_{n}\right\}$ is any sequence of positive numbers tending to 0 , then there is a noncyclic function $f=\sum a_{n} z^{n}$ with

$$
\left|a_{n}\right| \leq \exp \left(-n \epsilon_{n}\right)(n \geq 0) \text {. }
$$

The next result gives another description of the subspaces $K_{f}$ that is useful. Recall that, by Theorem 2, if $f \in N$ then signum $f^{2} \in Q$.

Lemma. If $f \in N$ with inner factor $f_{i}$ and if

$$
\frac{z \operatorname{signum} f^{2}}{f_{i}}=\frac{\phi}{\psi} \quad(\text { for }|z|=1)
$$

where $\phi$ and $\psi$ are relatively prime inner functions, then $K_{f}=\left(\phi H^{2}\right)^{\perp}$.

We now discuss the analytic continuation of noncyclic vectors. Recall that a meromorphic function is said to be of bounded characteristic if it is equal to the quotient of two bounded analytic functions.

THEOREM 6. If $f \in N$, then there is a function $F$, meromorphic and of bounded characteristic in $1<|z| \leqq \infty$ having the same radial limits on $|z|=1$ as does $f$, and conversely.

The function $F$ could be called a pseudo-continuation of $f ; f$ need not be analytically continuable across any arc of the boundary, but if it is somewhere continuable then the continuation must coincide with $F$. 
COROLlARY. If a noncyclic vector $f$ can be continued analytically across one or more arcs of the boundary, then the continuation is singlevalued and analytic in $1<|z| \leqq \infty$.

Corollary. $\log (1-z)$ and $(1-z)^{1 / 2}$ are cyclic.

By the support of an inner function $\phi$ we mean the union of the support of the singular measure associated with $\phi$ and the limit points of the zeros of $\phi$. Thus the support is a closed subset of the boundary of the unit disc.

THEOREM 7. If $\phi$ is inner and if $f \in\left(\phi H^{2}\right)^{\perp}$ and the support of $\phi$ is a proper subset of the boundary, then $f$ can be continued analytically across the complement of the support of $\phi$.

THEOREM 8. If $f \in N$ and $\phi$ is an inner function, then the composed function $f \circ \phi$ is in $N$.

Sarason has shown that the converse of this theorem is also valid.

Let $P$ denote the projection of $L^{2}$ onto $H^{2}$.

Theorem 9. If $f \in L^{2}$, then $P f \in N$ if and only if $\bar{f}$ is equal, almost everywhere on $|z|=1$, to the quotient of two bounded analytic functions.

Corollary. If $h \in L^{\infty}$ and $|h|=1$ a.e., then $P h \in N$ if and only if $h \in Q$.

For $h \in L^{\infty}$ let $T_{h}$ denote the Toeplitz operator defined on $H^{2}$ by: $T_{h} f=P(h f)$.

CoRollary. If $h \in L^{\infty}$, then $T_{h} N \subset N$ if and only if $\bar{h}$ is the quotient of two bounded analytic functions.

Instead of regarding the elements of $\mathrm{H}^{2}$ as functions defined in the interior of the disc, or on the boundary, we may also regard them as square-integrable sequences of complex numbers (Taylor coefficients). This point of view leads to some results of a different nature.

THEOREM 10. If $F$ is an entire function of exponential type $<\pi$, and if $F \in L^{2}$ on the real axis, then $\{F(n)\}(n \geqq 0)$ is a cyclic vector.

The condition $\sum|F(n)|^{2}<\infty$ is automatic, even if the type of $F$ equals $\pi$.

THEOREM 11. If $p$ and $q$ are polynomials such that the sequence $\{p(n) / q(n)\}$ is in $l^{2}$, then this sequence is a cyclic vector.

THEOREM 12. If $F$ is an entire function with $\{F(n)\}(n \geqq 0)$ in $l$ and if 


$$
|F(z)| \leqq a \exp (b \sqrt{|s|})
$$

for some $a, b>0$ and all $z$, then $\{F(n)\}$ is noncyclic.

We also consider shift operators of higher multiplicities (finite or countably infinite), that is, multiplication by the independent variable on vector-valued $H^{2}$ spaces. We show that the backward shift always has a dense set of cyclic vectors, while the forward shift has no cyclic vectors when the multiplicity is greater than one.

Finally, the results for the scalar case carry over unchanged to the $H^{p}$ spaces $(1<p<\infty)$.

\section{BIBLIOGRAPHY}

1. K. Hoffman, Banach spaces of analytic functions, Prentice-Hall, Englewood Cliffs, N. J., 1962.

2. H. S. Shapiro, Weakly invertible elements in certain function spaces, and generators in $l_{1}$, Mich. Math. J. 11 (1964), 161-165.

UNIVERSITY OF MichigaN 